http://dx.doi.org/10.22319/rmcp.v10i1.4739

Artículo

\title{
Evaluación y análisis sensorial del Queso Bola de Ocosingo (México) desde la
} perspectiva del consumidor

Mónica Agudelo-López ${ }^{\mathrm{a}}$

Alfredo Cesín-Vargas ${ }^{b^{*}}$

Angélica Espinoza-Ortega ${ }^{c}$

Benito Ramírez-Valverde ${ }^{\mathrm{d}}$

${ }^{a}$ Universidad Autónoma Chapingo- CIESTAAM, Km. 38.5. Carretera México- Texcoco. CP 56230. Estado de México, México.

${ }^{\mathrm{b}}$ Universidad Nacional Autónoma de México-UAER, Coordinación de Humanidades, Av. Lázaro Cárdenas s/n esquina Felicitas del Río, Jiquilpan, CP 59510. Michoacán, México.

${ }^{\mathrm{c}}$ Universidad Autónoma del Estado de México- ICAR, Toluca, Estado de México. México.

${ }^{\mathrm{d}}$ Colegio de Postgraduados Campus-Puebla. Puebla, México.

*Autor de correspondencia: alfredo.cesin@gmail.com.

\section{Resumen:}

El objetivo fue analizar la aceptación y preferencia del consumidor por el Queso Bola de Ocosingo con tres tiempos de maduración: fresco, 21 días y 45 días. Se realizaron 90 encuestas en tres escenarios relacionados con la gastronomía: Gourmet Show, Feria Nacional de la Cultura Rural en Chapingo y Muestra Gastronómica. La información se analizó a través de pruebas estadísticas paramétricas y no paramétricas. Al contrastar las respuestas, con los escenarios de evaluación y las características socioeconómicas de los panelistas, se encontró que aquellos que reportaron ingreso y nivel educativo más bajos, percibieron mejor las características visuales, prefiriendo los quesos más frescos; mientras que los panelistas con ingreso y nivel educativo más altos, apreciaron mejor los atributos asociados al aroma y el sabor del queso más maduro. El Queso Bola de Ocosingo comparte características productivas y culturales con otros quesos artesanales mexicanos, por lo que se espera contribuir para preservarlos como parte del patrimonio gastronómico del país. 
Palabras clave: Aceptabilidad, Alimentos diferenciados, Ferias gastronómicas, Panelistas no entrenados, Preferencia.

Recibido: 04/01/2018

Aceptado: 21/02/2018

\section{Introducción}

En las últimas décadas se han incrementado los movimientos que buscan conservar y promover la producción y consumo de productos locales. Esto responde parcialmente, al miedo que genera en el consumidor la excesiva industrialización y el desconocimiento del origen de los alimentos, que ha llevado a los consumidores a relacionarse con atributos externos como precios, presentaciones, etiquetas y sellos ${ }^{(1,2)}$.

Adicionalmente, diversos autores mencionan la importancia de recuperar la confianza en los alimentos a través del retorno a lo local, protegiendo la diversidad cultural contenida en los $\operatorname{mismos}^{(3,4)}$. Así, está surgiendo un nuevo segmento de consumidores preocupados por la forma en que se producen los alimentos y con capacidad para gestionar su propia comida; y que, pueden contribuir a recobrar el significado tradicional de la relación productor-consumidor, por ejemplo, reactivando los mercados locales, las ferias y otro tipo de eventos gastronómicos como escenarios para la valorización de los productos ${ }^{(1,4-6)}$.

La mayoría de trabajos que refieren al estudio de los cambios que han tenido los consumidores se han realizado principalmente en países europeos ${ }^{(7-10)}$; sin embargo, actualmente pareciera que también en Latinoamérica el consumidor está cambiando y se desconocen sus características y principales motivos de preferencia, porque los estudios existentes en países en vías de desarrollo han considerado principalmente los efectos de la inserción de la agricultura en el mercado mundial con sus implicaciones sobre las formas tradicionales de producción y los cambios sobre la población locales, especialmente en la dieta ${ }^{(1)}$. Esta situación que remite a la necesidad de estudiar los gustos y preferencias que tiene el consumidor por los productos locales, entre ellos, los quesos artesanales mexicanos.

En México se han identificado alrededor de 40 quesos diferentes, algunos elaborados en zonas marginadas del país y que todavía conservan sus formas de producción artesanal, cuya comercialización se realiza principalmente en mercados locales, cumpliendo un papel importante en el desarrollo económico de los territorios ${ }^{(11)}$. 
El queso bola (QBO) es elaborado por diez queseros artesanales en Ocosingo, en el estado de Chiapas, en el sureste del país. Es una bola de queso ácido de doble crema de leche cruda de vaca, y cubierto con un doble forro de queso, descremado. Tradicionalmente el proceso inicia con la recepción de la leche, a la cual se le agrega crema para hacer más suave la textura interna del queso; después se cuaja y desuera; la pasta resultante se madura en mantas, por mínimo 21 días antes del forrado, las cuales se cuelgan y se cambian periódicamente para evitar la contaminación del producto. Transcurrido el plazo de maduración, se elabora una bola de queso que es forrada con dos capas de otro queso descremado, de pasta hilada, cuya función es proteger el relleno, favoreciendo su comercialización a temperatura ambiente, sin contaminarse en el interior ${ }^{(11,12)}$.

La competencia con quesos industrializados de otro tipo y con menor precio, sumado a la reputación y demanda del queso bola original, han ocasionado cambios negativos en el proceso, específicamente en la reducción del tiempo de acidificación de la cuajada, aumentando la rotación de inventarios que repercute en la reducción de costos financieros, y mayores ganancias. Es por ello que en el mercado local se encuentran tres tipos de queso, uno fresco, el tradicional de 21 días y otro de 45 días, pero que no están claramente diferenciados ni en etiqueta (especialmente en los quesos frescos y de 21 días) ni en precio. Esto representa una pérdida del patrimonio agroalimentario, porque en el proceso de elaboración del queso fresco, la cuajada no se deja acidificar durante el tiempo que tradicionalmente se ha considerado, respetando el saber hacer y garantizando un producto inocuo; algo que también constituye un riesgo a la salud del consumidor, al tratarse de un queso, que independiente de los días de acidificación de la cuajada, es elaborado con leche cruda.

Se han realizado estudios con quesos de más de 21 días de acidificación de la cuajada que demuestran su calidad microbiológica ${ }^{(12,13)}$; pero el queso fresco no ha sido estudiado, lo que sugiere la necesidad, en un principio, de determinar si existe un consumidor que lo valore, y de acuerdo a esto identificar necesidades futuras de investigación que conlleven a su conservación como queso artesanal, además de garantizar su inocuidad. Por lo anterior, el objetivo de este trabajo fue conocer la aceptación y preferencia del consumidor por el queso bola de Ocosingo con tres tiempos de maduración, fresco, de 21 y 45 días, y analizar las implicaciones de esa preferencia.

\section{Material y métodos}

\section{Selección de panelistas y escenarios de evaluación}

El estudio se realizó con consumidores (panelistas no entrenados), considerados como idóneos por mostrar respuestas espontáneas, rápidas y no condicionadas al resultado de entrenamientos 
previos $^{(14-16)}$. Los panelistas fueron asistentes de tres eventos gastronómicos: i) Gourmet Show (GS), se realiza cada año en el World Trade Center de la ciudad de México, reuniendo a proveedores de productos de calidad para la cocina con consumidores potenciales; ii) Feria Nacional de la Cultura Rural en la Universidad Autónoma Chapingo (FCR), un evento anual que reúne a productores provenientes de todo el país, que elaboran productos locales, artesanías y alimentos para su exhibición y venta, también destinan un espacio para la venta de alimentos preparados representativos de cada estado, funcionando como un corredor gastronómico y, iii) Muestra Gastronómica (MG) del Instituto de Ciencias Agropecuarias y Rurales (ICAR,) de la Universidad Autónoma del Estado de México, realizada para conmemorar el día internacional de la alimentación y en la que confluyen estudiantes, docentes y personal administrativo de la institución. La elección de los escenarios fue por conveniencia, aprovechando la facilidad de acceso para realizar las pruebas sensoriales.

Para este tipo de estudios se recomienda evaluar entre 50 y 500 panelistas, los cuales generalmente son reclutados por ser usuarios, o porque están familiarizados con los productos a evaluar ${ }^{(15,17)}$. Se entrevistaron a 90 panelistas, distribuidos en los tres escenarios descritos: $34.4 \%$ en el GS, $30 \%$ en la FCR y $35.6 \%$ en la MG; que si bien, la mayoría, no conocían con antelación el queso, se eligieron porque al asistir a este tipo de eventos, pueden valorar los productos locales elaborados artesanalmente. Además, el queso es poco conocido a nivel nacional, por lo que se espera que las respuestas espontáneas de panelistas no entrenados, al no recibir entrenamientos previos, sean más parecidas a las del promedio de la población ${ }^{(15)}$.

\section{Muestras evaluadas}

Se utilizaron muestras de QBO elaboradas en una misma quesería, para evitar sesgos relacionados con el proceso. Los quesos sometidos a las pruebas fueron: i) queso fresco con tres días de acidificación de la cuajada antes del forrado, y en total con 17 a 19 días de elaborado (en adelante queso fresco); ii) queso con 21 días de acidificación de la cuajada previo al forrado y en total con 35 a 37 días de elaborado (en adelante queso de 21 días); y iii) queso con 45 días de acidificación de la cuajada antes del forrado y en total con 59 a 61 días de elaborado (en adelante queso de 45 días).

Las muestras se distribuyeron en un plato, codificadas aleatoriamente, para evitar que fueran asociadas con los días de maduración y se dispuso de agua, pan y fruta (manzanas verdes) para la limpieza de las papilas gustativas de los panelistas antes de iniciar la evaluación y entre muestras. 


\section{Pruebas realizadas}

Se aplicaron dos pruebas, una de aceptación y otra de preferencia. La primera indica el nivel de agrado de las características sensoriales de cada muestra, acorde con una escala de valoración, mientras que en la segunda se elige la muestra de mayor agrado ${ }^{(15)}$. Al final de las pruebas, se solicitó a los panelistas información de procedencia, género, edad e ingreso para facilitar su estratificación.

En la evaluación de aceptación se consideraron diez atributos: apariencia externa e interna, color externo e interno, textura en mano, olor, textura en boca, sabor, retrogusto y precio; se utilizó una escala ordinal de cinco puntos (1: no me gusta; 2: me gusta un poco; 3: me es indiferente; 4: me gusta y 5: me gusta mucho), acompañada de una escala visual como estrategia para agilizar el proceso, y evitar, en caso de existir panelistas sin educación formal, un factor de discriminación ${ }^{(18)}$.

En la segunda prueba, los panelistas indicaron el orden de preferencia de las muestras evaluadas. Al final de las pruebas, los panelistas fueron informados de las características de cada queso y de sus condiciones de elaboración, para responder a la disposición a comprar y a pagar un valor diferencial por el queso preferido (considerando un rango de $\pm 60 \%$ del precio base por pieza de $300 \mathrm{~g})$.

\section{Análisis estadístico}

Para validar la fiabilidad de las preguntas realizadas se utilizó el coeficiente alfa de Cronbach, tomando en cuenta los 30 atributos que forman las tres pruebas. El resultado mostró un valor de 0.935, por lo que se consideró que el cuestionario era fiable para captar la información sobre las características de los quesos. Este coeficiente se obtuvo mediante la utilización del paquete estadístico SPSS versión 16.

Para el análisis de los datos se aplicaron diversas técnicas estadísticas, utilizando el paquete estadístico Infostat ${ }^{(19,20)}$ : Para la comparación de los atributos entre quesos y dado que cada panelista, de los tres escenarios seleccionados, probó las tres muestras y dio su opinión respecto a cada atributo, se consideraron como muestras relacionadas, analizadas mediante la prueba de Friedman. Para facilitar la presentación de resultados, los atributos se clasificaron en: visuales (apariencia externa e interna, color externo e interno) y otros (textura en mano, olor, textura en boca, sabor y retrogusto). La percepción del precio se presentó por separado, dado que no se considera un atributo propiamente sensorial, pero su inclusión es fundamental para tener una visión más completa de la evaluación ${ }^{(21,22)}$. 
Para comparar la diferencia entre los tres escenarios de evaluación respecto a los atributos de cada uno de los quesos, se empleó la prueba de Kruskal Wallis. Para buscar las preferencias respecto a los quesos, se utilizaron pruebas estadísticas paramétricas y no paramétricas de acuerdo a la escala de medición de la variable. Las variables cuantitativas fueron comparadas a través de análisis de varianza, con prueba de Tukey para la separación de medias y las nominales con la prueba de Ji cuadrada. Finalmente, para buscar diferencias en la disposición a pagar un valor diferente al precio base, se usó la prueba de Kruskal Wallis para comparar los resultados por escenario de evaluación.

\section{Resultados y discusión}

\section{Perfil socioeconómico de los panelistas}

Los asistentes del GS son en su mayoría de la Ciudad de México y a diferencia de los otros dos grupos, presentan el nivel de escolaridad más alto y también mayor ingreso; en el grupo de la FCR predominaron personas de diferentes partes del país, y es el grupo con menor escolaridad; los asistentes de la MG fueron en su mayoría estudiantes, provenientes del Estado de México y los de menor edad promedio (Cuadro 1).

Cuadro 1: Características del perfil del consumidor por escenario de evaluación

\begin{tabular}{rccccc}
\hline $\begin{array}{c}\text { Variable/ escenario } \\
\text { de evaluación }\end{array}$ & Gourmet Show & $\begin{array}{c}\text { Feria de la } \\
\text { Cultura } \\
\text { Rural }\end{array}$ & $\begin{array}{c}\text { Muestra } \\
\text { Gastronómica }\end{array}$ & Estadístico & $\boldsymbol{P}$ \\
\hline Lugar de origen, \% & & & & $\mathrm{X}^{2}=26.722$ & $<.0001$ \\
Ciudad de México & 48.4 & 26 & 3.1 & & \\
Estado de México & 38.7 & 33.3 & 81.3 & & \\
Otros estados & 12.9 & 40.7 & 15.6 & & \\
Edad, años & $32.5 \pm 11.1$ (ab) & $34.4 \pm 17.2(\mathrm{~b})$ & $26.6 \pm 6.3(\mathrm{a})$ & $\mathrm{F}=3.43$ & $<.037$ \\
Género, \% & & & & $\mathrm{X}^{2}=0.525$ & .776 \\
Masculino & 51.6 & 51.9 & 43.7 & & \\
Femenino & 48.4 & 48.1 & 56.3 & $\mathrm{~F}=27.169$ & $<.001$ \\
Escolaridad, años & $17.3 \pm 3.4(\mathrm{~b})$ & $12.4 \pm 2.8(\mathrm{a})$ & $16.2 \pm 2.5(\mathrm{~b})$ & $\mathrm{X}^{2}=36.865$ & $<.001$ \\
Ocupación, \% & & 7.4 & 9.4 & & \\
Profesionales & 30 & 25.9 & 0 & & \\
Independientes & 20 & 25.9 & 0 & & \\
Empleados & 16.7 & 40.7 & 90.6 & & \\
Estudiantes & 33.3 & $442 \pm 347$ (a) & $415 \pm 243$ (a) & $\mathrm{X}^{2}=8.98$ & \\
Ingreso mensual* & $1,077 \pm 995$ (b) & & & & \\
\hline
\end{tabular}

* Dólares. Tipo de cambio: 18.492. Fuente: Banco de México (http://www.banxico.org.mx/dyn/portal-mercadocambiario/index.html). Consultado el 18 de julio de 2016. a,b Medias con letra en común no son diferentes $(P>0.05)$. 


\section{Percepción de los atributos por tipo de queso y por escenario de evaluación}

Para identificar la percepción general del consumidor por tipo de queso, a través de la prueba de Friedman, se observa que no hay diferencias estadísticas significativas por tipo de queso (Cuadro 2). Es importante mencionar que el QBO, al ser un producto sui géneris dentro de los quesos artesanales mexicanos, posee características que lo diferencian de otros tipos de queso del país ${ }^{(11)}$, y al tratarse de un queso llamativo para los panelistas, influyó en la evaluación positiva de todos sus atributos.

Cuadro 2: Percepción de los atributos por tipo de queso

\begin{tabular}{|c|c|c|c|c|c|}
\hline \multirow[b]{2}{*}{ Atributo } & \multicolumn{3}{|c|}{ Promedio* } & \multirow[b]{2}{*}{$\mathbf{T}^{2}$} & \multirow[b]{2}{*}{$P$} \\
\hline & $\begin{array}{l}\text { Queso } \\
\text { fresco }\end{array}$ & $\begin{array}{c}\text { Queso de } 21 \\
\text { días }\end{array}$ & $\begin{array}{c}\text { Queso de } 45 \\
\text { días }\end{array}$ & & \\
\hline Atributos visuales & 3.95 & 4.01 & 3.92 & 0.39 & .6789 \\
\hline Otros atributos & 3.53 & 3.48 & 3.43 & 1.14 & .3208 \\
\hline $\begin{array}{l}\text { Percepción del } \\
\text { precio }\end{array}$ & 3.29 & 3.41 & 3.44 & 1.35 & .2619 \\
\hline
\end{tabular}

* La escala de medición es de tipo ordinal, pero se utiliza el promedio para mostrar la tendencia de la medición del atributo.

En general, se observa que los atributos visuales fueron mejor evaluados, reflejando en primera instancia que se trata de consumidores que dan mayor importancia a las características que les son más fácilmente perceptibles; situación que puede ser reflejo del modelo global empleado para el abasto de alimentos, basado en el sistema de símbolos y señales, diseñado para cubrir el distanciamiento entre productor y consumidor ${ }^{(1,23)}$. Por otro lado, la ausencia de diferencias entre muestras podría atribuirse a, que al tratarse de un queso no convencional y llamativo (sólo el 15.6 \% lo conocía con antelación), sea congruente con la evaluación positiva de todos los atributos; pero al no tener experiencia como panelistas, no lograron detectar las diferencias sensoriales entre las muestras. Esto coincidió con otro estudio en el que evaluaron sensorialmente quesos de cabra con diferentes días de maduración, concluyendo que los consumidores que no estaban familiarizados con el consumo de quesos con olores y sabores intensos, no podían reconocer con facilidad las diferencias sensoriales entre muestras ${ }^{(24)}$.

Mediante la prueba de Kruskal Wallis se compararon los atributos por escenario de evaluación y tipo de queso, encontrándose diferencias estadísticas significativas para los atributos visuales en las tres muestras de queso, mientras que los otros atributos y la percepción del precio, sólo fueron diferentes en el queso de 45 días (Cuadro 3). 
Cuadro 3: Comparación de los atributos sensoriales por escenario de evaluación y tipo de queso

\begin{tabular}{|c|c|c|c|c|c|}
\hline \multirow[t]{2}{*}{ Atributo } & \multicolumn{3}{|c|}{ Promedio* } & \multirow[b]{2}{*}{$\mathrm{H}$} & \multirow[b]{2}{*}{$P$} \\
\hline & $\begin{array}{c}\text { Gourmet } \\
\text { Show }\end{array}$ & $\begin{array}{c}\text { Feria de la } \\
\text { Cultura Rural }\end{array}$ & $\begin{array}{c}\text { Muestra } \\
\text { Gastronómica }\end{array}$ & & \\
\hline \multicolumn{6}{|l|}{ Queso fresco } \\
\hline Atributos visuales & 3.86 (a) & 3.68 (a) & 4.27 (b) & 8.98 & .0105 \\
\hline Otros atributos & 3.48 & 3.56 & 3.55 & 0.09 & .9547 \\
\hline $\begin{array}{r}\text { Percepción del } \\
\text { precio }\end{array}$ & 3.13 & 3.15 & 3.56 & 2.37 & .279 \\
\hline \multicolumn{6}{|l|}{ Queso de 21 días } \\
\hline Atributos visuales & $3.99(a b)$ & 3.72 (a) & $4.28(\mathrm{~b})$ & 6.77 & .0315 \\
\hline Otros atributos & 3.63 & 3.32 & 3.48 & 1.07 & .5824 \\
\hline $\begin{array}{r}\text { Percepción del } \\
\text { precio }\end{array}$ & 3.58 & 2.96 & 3.63 & 5.03 & .0652 \\
\hline \multicolumn{6}{|l|}{ Queso de 45 días } \\
\hline Atributos visuales & $4.04(\mathrm{ab})$ & 3.64 (a) & $4.11(\mathrm{~b})$ & 6.07 & .0457 \\
\hline Otros atributos & 3.93 (b) & 3.04 (a) & 3.27 (a) & 11.25 & .0035 \\
\hline $\begin{array}{r}\text { Percepción del } \\
\text { precio }\end{array}$ & 3.97 (b) & 2.93 (a) & $3.38(a b)$ & 10.15 & .0046 \\
\hline
\end{tabular}

"La escala de medición es de tipo ordinal, pero se utiliza el promedio para mostrar la tendencia de la medición del atributo.

ab Medias con letra en común no son diferentes $(P>0.05)$.

Los asistentes de la FCR tuvieron las valoraciones más bajas en todos los quesos y, al igual que los de la MG, dieron mayor importancia a los atributos visuales de todos los quesos, mientras que los del GS valoraron mejor los otros atributos y el precio en el queso de 45 días. Es importante notar que los panelistas del GS mostraron una evaluación más consistente, al ir de menos a más en las valoraciones de los quesos a medida que avanzaban en la maduración (Cuadro 3). En los otros dos casos las tendencias no fueron tan claras, aunque se observa un comportamiento contrario, las valoraciones más positivas las dieron para el queso fresco y disminuyen a medida que va aumentando la maduración. Las diferencias encontradas en la percepción de atributos en el queso con diferentes días de maduración, pueden explicarse en función de las características de los panelistas y de la posibilidad que tienen para acceder regularmente a este tipo de productos.

El grupo de la FCR fue el más diverso y con la escolaridad más baja, mientras que los de la MG, en su mayoría estudiantes pero más jóvenes; en ambos grupos se identificó el ingreso más bajo, comparados con los del GS (Cuadro 1); es comprensible que los participantes de los grupos con bajo ingreso, valoren mejor las características de los quesos más frescos, dado que son los más ofertados en el país y generalmente, son más baratos ${ }^{(25,26)}$. Además, en la MG, pese que fueron estudiantes de carreras afines con la valorización de productos locales, pero al ser más jóvenes, no 
cuentan con la experiencia suficiente para distinguir y valorar un producto por sus condiciones intrínsecas, por lo que dieron mayor importancia a los atributos visuales. Por otra parte, el perfil de los asistentes del GS (al tener mayor escolaridad e ingreso), coincide con el tipo de consumidores que buscan alimentos diferenciados y de calidad. Son personas que aprecian el "buen comer" y buscan experiencias sensoriales que no ofrecen productos de elaboración masiva (que también pueden estar elaborados con materias primas de buena calidad), adquiriendo parte de su comida en mercados exclusivos de productos especiales o de manufactura artesanal. Probablemente, el mayor contacto de estos consumidores con productos diferenciados, les permite valorarlos más allá de la apariencia visual.

Dado lo anterior, los resultados sugieren la existencia de dos tipos de consumidores, aquellos que motivan sus elecciones basados en atributos intrínsecos, asociadas al grado de conocimiento y experiencia sobre el producto o similares, coincidiendo con el perfil de los asistentes al GS; y también otros que consideran aspectos visuales, entre ellos la apariencia del producto, como una codificación previa para decidir la compra, propio de los resultados encontrados para la MG y la $\mathrm{FCR}^{(6,22)}$.

Respecto a la percepción del precio, se encontró diferencia estadística significativa en el queso de 45 días (Cuadro 3). Es comprensible que los asistentes del GS, mientras valoraron menor el precio del queso fresco, percibieron con agrado el precio para el queso de 45 días, al tener mayor riqueza sensorial, y que en el mercado se vende al mismo precio que el fresco. Si cuestan lo mismo, van a aceptar con mejor disposición el precio del queso con características sensoriales más intensas; ocurriendo todo lo contrario con los panelistas de la FCR y de la MG, que percibieron mejor el precio del queso fresco y de 21 días, respectivamente, en comparación con el de 45 días y que indica que al no estar acostumbrados al consumo de productos con aromas y sabores fuertes, consideran que su precio es alto, así cuesten lo mismo. Un componente importante de la evaluación era determinar si los resultados de la percepción de los atributos, coincidía con la elección de preferencia realizada por los panelistas.

\section{Comparativo de la prueba de preferencia por escenario de evaluación}

Los resultados de preferencia de los panelistas, sugieren la existencia de un consumidor particular para cada tipo de queso; adicionalmente, mediante la prueba de Ji cuadrada se encontraron diferencias estadísticas por escenario de evaluación $\left(\chi^{2}=8.121 ; P=0.087\right)$, indicando algunas preferencias, especialmente en el GS y la FCR (Figura 1). En el GS la mayoría de panelistas prefirieron el queso de 45 días, mientras que en la FCR la mayor preferencia fue por el queso fresco. En la MG no hubo una preferencia marcada hacia algún tipo de queso. 
Figura 1: Preferencia general y por escenario de evaluación

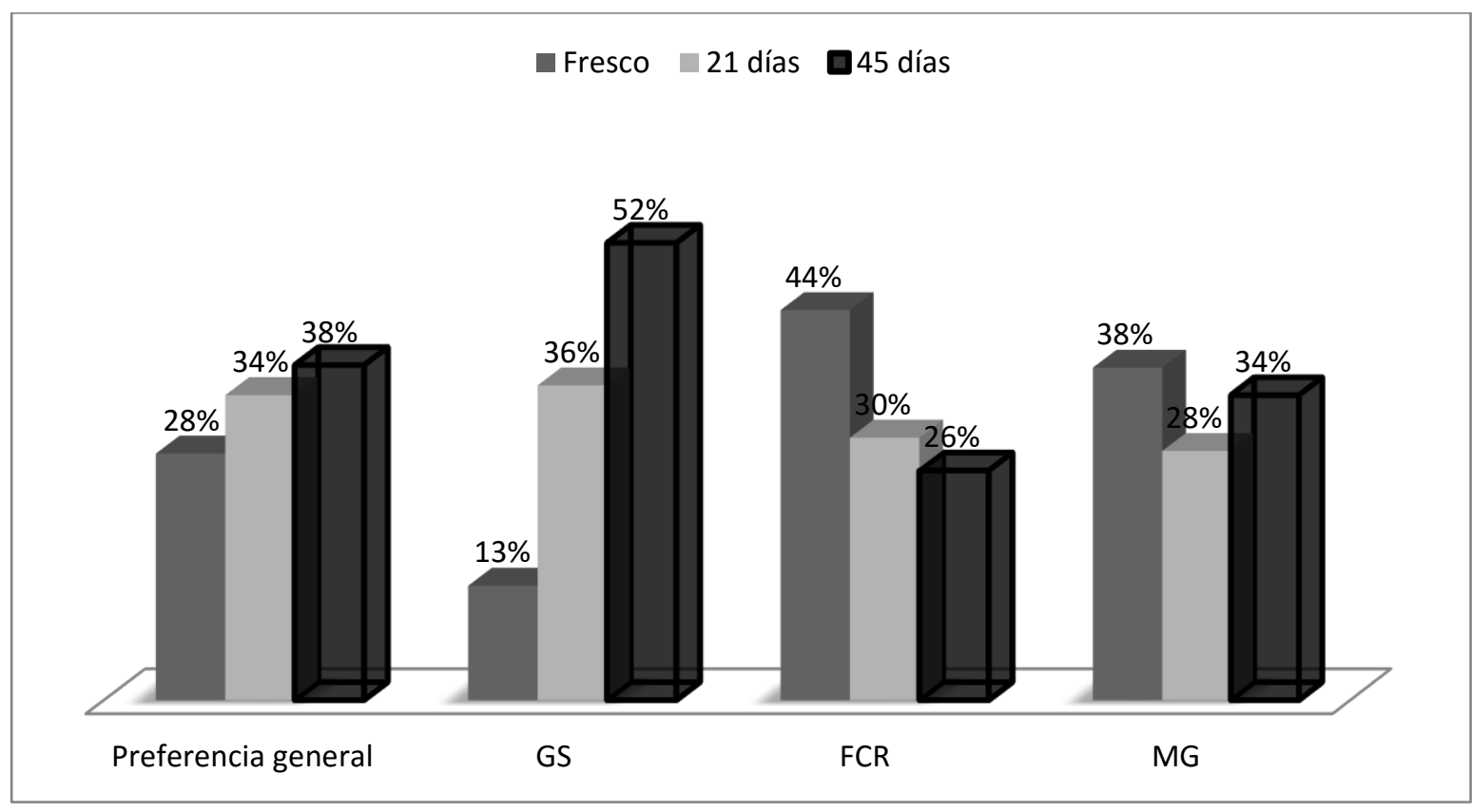

GS= Gourmet Show; FCR= Feria Nacional de la Cultura Rural; MG= Muestra Gastronómica.

La preferencia de los consumidores se puede explicar también considerando las características demográficas, accesibilidad y frecuencia de contacto con productos diferenciados, y por la misma influencia que pudieron tener los escenarios de evaluación sobre las experiencias individuales de los panelistas ${ }^{(2)}$. En el Cuadro 4 se presentan las características del perfil de los consumidores en función del queso preferido, encontrándose que la preferencia por un tipo de queso particular, estuvo relacionada especialmente con la escolaridad y el ingreso.

Cuadro 4: Características del perfil del consumidor por tipo de queso preferido

\begin{tabular}{cccccc}
\hline $\begin{array}{c}\text { Variable/Tipo } \\
\text { de queso } \\
\text { preferido }\end{array}$ & $\begin{array}{c}\text { Queso } \\
\text { fresco }\end{array}$ & $\begin{array}{c}\text { Queso de } \\
\text { 21 días }\end{array}$ & $\begin{array}{c}\text { Queso de } \\
\text { 45 días }\end{array}$ & Estadístico & $\boldsymbol{P}$ \\
\hline $\begin{array}{r}\text { Lugar de origen, \% } \\
\text { Ciudad de } \\
\text { México }\end{array}$ & 24 & 22.6 & 29.4 & & \\
$\begin{array}{c}\text { Estado de } \\
\text { México }\end{array}$ & 44 & 51.6 & 58.8 & & .424 \\
$\begin{array}{c}\text { Otros estados } \\
\text { Edad, años }\end{array}$ & $31.12 \pm 10.9$ & $27.97 \pm 12.3$ & $33.62 \pm 13.1$ & $\mathrm{~F}=1.723$ & .185 \\
Género, \% & & & & $\mathrm{X}^{2}=0.404$ & .834 \\
\hline
\end{tabular}




\begin{tabular}{rccccc}
\hline Masculino & 48 & 45.2 & 52.9 & & \\
Femenino & 52 & 54.8 & 47.1 & & \\
Escolaridad, & $14.67 \pm 3.7$ & $14.71 \pm 3.2$ & $16.82 \pm 3.3$ & $\mathrm{~F}=4.059$ & .021 \\
años & $(\mathrm{a})$ & $(\mathrm{ab})$ & $(\mathrm{b})$ & & \\
$\begin{array}{r}\text { Ocupación, \% } \\
\text { Profesionales }\end{array}$ & 16 & 6.5 & 24.2 & & \\
Actividades & 16 & 16 & 12.1 & & \\
independientes & & & & & \\
$\quad$ Empleados & 20 & 9.7 & 12.1 & & \\
$\quad$ Estudiantes & 48 & 67.7 & 51.5 & & \\
ingreso & $463 \pm 255$ & $467 \pm 471$ & $945 \pm 948$ & $\mathrm{~F}=4.617$ & .013 \\
mensual* & (a) & (a) & (b) & & \\
\hline
\end{tabular}

* Dólares. Tipo de cambio: 18.492. Fuente: Banco de México (http://www.banxico.org.mx/dyn/portal-mercadocambiario/index.html). Consultado el 18 de julio de 2016.

El análisis de varianza mostró diferencias estadísticas significativas en la preferencia por el queso de 45 días, indicando que las personas con mayor nivel académico e ingreso, prefirieron los quesos con mayor tiempo de maduración. Además, los resultados de preferencia por escenario de evaluación coincidieron con los de las pruebas de aceptación, permitiendo inferir la existencia de dos tipos de consumidores: en el primero, la aceptación y preferencia por quesos más frescos se dio en la población de menor ingreso, baja escolaridad y en el escenario en que la gastronomía hace parte de un evento asociado a la cultura en general (representado por los asistentes de la FCR); mientras que los del segundo, la aceptación y preferencia por quesos más maduros se dio en la población con ingreso más alto, mayor escolaridad y en escenarios cuya esencia es la actividad gastronómica, por tanto, más especializados (asistentes del GS, principalmente). Los panelistas de la MG, al tener una preferencia menos marcada hacia una muestra particular de queso, se distribuyeron entre estos dos tipos.

Dado que las elecciones por determinados alimentos están influenciadas también por las experiencias propias del consumidor ${ }^{(22)}$, la elección del primer grupo, afín al consumo de quesos frescos, coincide con aquellos consumidores que prefieren quesos más suaves porque son los que comúnmente encuentran en el mercado ${ }^{(24)}$. Los consumidores de la FCR, por la naturaleza de la feria, probablemente comparten características similares al promedio de consumidores del país, a los cuales les resulta más fácil el consumo de quesos frescos, debido a su menor precio y mayor accesibilidad $^{(26)}$, mientras que el consumo de quesos maduros están reservados a algunas regiones del país donde son producidos, haciendo parte de sus tradiciones, y para el mercado gourmet de las grandes ciudades donde las ferias especializadas, juegan un papel clave.

Asimismo, los escenarios de evaluación, como parte de un contexto social que produce expectativas y experiencias individuales, pudieron influir en los resultados encontrados, porque además contribuyen con la codificación previa, aportando información indirecta de la calidad 
esperada de los productos que allí se ofrecen ${ }^{(2,27)}$. Esto permite explicar las razones por las que los consumidores asistentes al GS valoraron mejor los atributos intrínsecos y prefirieron el queso con mayor tiempo de maduración. Al asistir a este tipo de actividades donde la gastronomía diferenciada es la esencia del evento, esperan encontrar productos con gran riqueza sensorial; mientras que espacios como la FCR, al ser más generales y diversos (espacio en el que se ofrece eventos culturales, feria del libro, gastronomía y artesanías), la preferencia de los consumidores estuvo asociada al consumo frecuente de quesos frescos. Por su parte, en la MG, donde hubo similar aceptación por los tres tipos de queso, su elección pudiera relacionarse con la mayor afluencia de estudiantes relacionados con turismo, gastronomía y territorio, de ahí su grado de sensibilidad hacia los productos locales, pero sin el conocimiento necesario para percibir mejor las diferencias entre muestras.

Los resultados aportados por la percepción de los atributos y preferencia de los quesos, reflejan aspectos importantes para ser analizados como áreas de oportunidad que permitan mejorar las estrategias de mercado del QBO, en función de sus días de maduración y considerando además, el territorio de producción, la plataforma de ferias agroalimentarias y eventos gastronómicos, como escenarios importantes para la valorización de productos locales en México ${ }^{(28)}$.

El queso de 45 días, al ser preferido por un público más exclusivo, permite dimensionar el potencial que tiene para cautivar este tipo de consumidores en el territorio donde el queso es producido, aprovechando el municipio de San Cristóbal de las Casas como destino turístico de proximidad con Ocosingo ${ }^{(28)}$. Los otros dos grupos, más afines a valorar el queso por sus atributos visuales y más frescos, pero con menor ingreso, podrían reflejar la mayor parte de la población del país, a quien puede ofrecerse un queso, con menor tiempo de maduración, pero también más barato y que se constata en la disposición a pagar por el queso preferido.

\section{Intención de compra y disposición a pagar un precio diferente por el queso preferido}

Antes de suministrar información de los quesos a los panelistas, la percepción del precio fue uno de los atributos que recibió la más baja calificación (Cuadro 3). Algunos autores establecen que cuando se provee información relacionada con el producto, la imagen hacia el mismo cambia, reflejándose además, en la disposición a la compra y a pagar un valor preferencial ${ }^{(21,29)}$. Esto se vio reflejado al preguntarle a los panelistas por la intención de comprar el queso preferido, después de que se les proporcionara información relativa a la forma de elaboración del queso. En los tres escenarios hubo respuestas positivas, el $93.5 \%$ de los entrevistados del GS, $87 \%$ de la MG y 74 $\%$ de la FCR comprarían el queso; sin embargo, los del GS estarían dispuestos a pagar más de lo que cuesta el queso, los de la MG mostraron una disposición a pagar más cercana al precio base y 
los de la FCR, pagarían menos por el queso. El análisis de resultados se hizo mediante la prueba de Kruskal Wallis (Cuadro 5), encontrándose diferencias estadísticas significativas al 10\% en la disposición a pagar por los quesos fresco y de 21 días, y altamente significativa para el queso de 45 días.

Cuadro 5: Comparación de la disposición a pagar por el queso preferido entre los escenarios de evaluación

\begin{tabular}{cccccc}
\hline \multirow{2}{*}{$\begin{array}{c}\text { Disposición a } \\
\text { pagar }\end{array}$} & \multicolumn{2}{c}{ Promedio (proporción sobre el precio base)* } & & \multirow{2}{*}{ H } & $\boldsymbol{P}$ \\
\cline { 2 - 5 } & $\begin{array}{c}\text { Gourmet } \\
\text { Show }\end{array}$ & $\begin{array}{c}\text { Feria de la } \\
\text { Cultura Rural }\end{array}$ & $\begin{array}{c}\text { Muestra } \\
\text { Gastronómica }\end{array}$ & & \\
\hline Queso fresco & 0.15 & -0.05 & -0.01 & 4.77 & .0840 \\
Queso de 21 días & 0.12 & -0.08 & 0.00 & 4.76 & .0833 \\
Queso de 45 días & $0.18(\mathrm{~b})$ & -0.15 (a) & $-0.04(\mathrm{a})$ & 11.91 & .0022 \\
\hline
\end{tabular}

*La escala de medición es de tipo ordinal, pero se utiliza el promedio para mostrar la tendencia de la medición del atributo.

a,b Medias con letra en común no son diferentes $(P>0.05)$.

Al observar los resultados del Cuadro 5, se puede inferir que la disposición a pagar un sobreprecio, estuvo relacionada con el poder adquisitivo de los panelistas, también puede atribuirse al nivel educativo y con la posibilidad que tienen para acceder a productos con calidad diferenciada, dado que los del GS fueron los únicos dispuestos a pagar un valor adicional por cualquiera de los tres tipos de queso y que, mientras están dispuestos a pagar más por el queso de 45 días (18 \% adicional, en promedio), en los otros dos escenarios, donde se registró el menor ingreso (Cuadro 1), manifestaron la disposición de pago, por el mismo queso, por debajo del precio base.

Si bien el precio y el nivel de ingreso influyen en la decisión de compra, otros factores, intrínsecos del producto, podrían tener una mayor influencia sobre la decisión, pero esto implica que el consumidor tenga la posibilidad de conocer a fondo el producto. Diversos trabajos establecen que el sabor es el atributo más importante para determinar la calidad y la disposición a comprar un alimento local, pero sólo está disponible cuando se vive la experiencia sensorial, mientras que otros factores como precio, marca, presentación y el hecho de que sea artesanal o local, entran en juego cuando no se conoce nada más del producto ${ }^{(2,30,31)}$. Después de que los panelistas eligieron el queso preferido y manifestaron intención de compra, se indagó por las principales motivaciones para comprarlo, y se encontró que el sabor fue el atributo más importante para $55.4 \%$ de los panelistas, $20.7 \%$ porque lo consideró único y artesanal, $24 \%$ por factores como presentación, curiosidad y por ser un producto local, y sólo $6.5 \%$ lo comprarían por el precio. Lo que sugiere que cuando los panelistas participaron en la evaluación sensorial, el sabor fue más importante que el precio, pero que la posibilidad de pagar un valor adicional estuvo condicionada por variables socioeconómicas y por las experiencias del individuo. 


\section{Conclusiones e implicaciones}

El conocimiento de los gustos y preferencias del consumidor, proporcionado por estudios de este tipo, permitirá a los productores acceder a mercados especializados más rentables y que contribuyan al mantenimiento de las tradiciones, con el queso bola de más de 21 días de maduración y con cualidades organolépticas de mayor riqueza, valoradas en el mercado gourmet. Por otro lado, y al existir un consumidor que valoró cada tipo de queso evaluado, se parte de la premisa que, al ser un queso elaborado con leche cruda, cada tipo tiene que cumplir con la calidad microbiológica para garantizar que su ingesta no dañe la salud del consumidor. Los quesos con más de 21 días de acidificación de la cuajada tienen más posibilidades de ser inocuos al lograr un pH más bajo, a diferencia del queso fresco cuya calidad microbiológica se desconoce, representando un riesgo para los consumidores. Además, alguna patología relacionada con el consumo de queso fresco, con deficiente calidad microbiológica, afectaría también el prestigio de los otros dos tipos de queso, sensorialmente más ricos e inocuos. Por lo tanto, debido a la tendencia a comercializar proporcionalmente mayor cantidad de queso bola fresco, es importante que en futuras investigaciones se determine el tiempo mínimo de acidificación de la cuajada que garantice la inocuidad del queso.

\section{Literatura citada:}

1. Díaz C, García I. La mirada sociológica hacia la alimentación: análisis crítico del desarrollo de la investigación en el campo alimentario. Política y Sociedad; 2014;51:15-49.

2. Hansen T. Understanding consumer perception of food quality: the cases of shrimps and cheese. British Food J 2005;107(7):500-525.

3. Tregear A, Arfini F, Belletti G, Marescotti A. Regional foods and rural development: the role of product qualification. J Rural Studies 2007;23:12-22.

4. Acampora T, Fonte M. Productos típicos, estrategias de desarrollo rural y conocimiento local. Revista Opera 2008;(7):191-212.

5. Fonte M. Knowledge, food and place. A way of producing, a way of knowing. Sociologia Ruralis 2008;48(3):200-222.

6. Viola M. Estudios sobre modelos de consumo: una visión desde teorías y metodologías. Rev Chilena Nutr 2008;35(2):93-99.

7. Almli VL, Verbeke W, Vanhonacker F, Næs T, Hersleth M. General image and attribute perceptions of traditional food in six European countries. Food Qua 1 Prefer 
2011;22:129-138.

8. Pieniak Z, Verbeke W, Vanhonacker F, Guerrero L, Hersleth M. Association between traditional food consumption and motives for food choice in six European countries. Appetite 2009;53(1):101-108.

9. Cerjak M, Haas R, Brunner F, Tomić M. What motivates consumers to buy traditional food products? Evidence from Croatia and Austria using word association and laddering interviews. British Food J 2014;116(11):1726-1747.

10. Vanhonacker F, Lengard V, Hersleth M, Verbeke W. Profiling european traditional food consumers. British Food J 2010;112(8):871-886.

11. Villegas A, Cervantes F, Cesín A, Espinoza A, Hernández A, Santos A, et al. Atlas de los quesos mexicanos genuinos. Biblioteca Básica de Agricultura; Colegio de Posgraduados; Universidad Autónoma Chapingo; Instituto Interamericano de Cooperación para la Agricultura; 2014.

12. López R, Hernández A, Villegas A, Santos A, Escobar M. Caracterización socio-técnica del queso bola de Ocosingo, Chiapas. Villegas A, Santos A, Hernández A, editors. Texcoco, Estado de México: Universidad Autónoma Chapingo; 2013.

13. Escobar-Ramirez M, Perez-Escalante D, Mejia-Ruiz F, Avila-Vega D, Arvizu-Medrano S, Nava G, et al. Microbiological profile of two artisanal mexican cheeses during manufacturing process. IAFP 2012 Abstracts. Vol. 75. Providence, Rhode Island: J Food Protect; 2012:136.

14. Ares G, Deliza R, Barreiro C, Giménez A, Gámbaro A. Comparison of two sensory profiling techniques based on consumer perception. Food Qual Pref 2010;21:417-426.

15. Drake M. Invited review: Sensory analysis of dairy foods. J Dairy Sci 2007;90(11):49254937.

16. Faye P, Brémaud D, Teillet E, Courcoux P, Giboreau A, Nicod H. An alternative to external preference mapping based on consumer perceptive mapping. Food Qual Pref 2006;17:604614.

17. Ramírez-Navas JS. Análisis sensorial: pruebas orientadas al consumidor. ReCiTeIA 2012;12:84-101.

18. Schiffman LG, Kanuk LL. Comportamiento del consumidor. Décima edición. México: Pearson Educación; 2010.

19. Di Rienzo J., Casanoves F, Balzarini M., Gonzalez L, Tablada M, Robledo CW. InfoStat. Software estadístico. Argentina: Grupo InfoStat, FCA, Universidad Nacional de 
Córdoba; 2008.

20. Balzarini M., González L, Tablada M, Casanoves F, Di Rienzo J, Robledo CW. InfoStat. Software estadístico: manual del usuario. Córdoba, Argentina: Editorial Brujas; 2008.

21. Di Monaco R, Di Marzo S, Cavella S, Masi P. Valorization of traditional foods: The case of Provolone del Monaco cheese. British Food J 2005;107(2):98-110.

22. Cayot N. Sensory quality of traditional foods. Food chemistry 2007;101:154-162.

23. Díaz C. Los debates actuales en la sociología de la alimentación. Rev Int Sociol 2005;(40):47-78.

24. Ryffel S, Piccinali P, Bütikofer U. Sensory descriptive analysis and consumer acceptability of selected Swiss goat and sheep cheeses. Small Ruminant Res 2008;79:80-86.

25. Cesín A, Aliphat M, Ramírez B, Herrera JG, Martínez D. Family milk and cheese production. A study in three communities in the Municipality of Tetlatlahuca in the State of Tlaxcala, México. Téc Pecu Méx 2007;45:61-76.

26. Jiménez-Guzmán J, Flores-Nájera A, Cruz-Guerrero AE, García-Garibay M. Use of an exopolysaccharide-producing strain of Streptococcus thermophilus in the manufacture of Mexican Panela cheese. LWT - Food Sci Technol 2009;42:1508-1512.

27. Costell E, Tárrega A, Bayarri S. Food acceptance: the role of consumer perception and attitudes. Chemosensory Percep 2010;3:42-50.

28. Agudelo-López MA, Cesín-Vargas A, Thomé-Ortíz H. Emblematic foods and tourism: the link between the Bola Cheese from Ocosingo and the regional tourism offer. Agr Sociedad Desarrollo 2016;13:131-149.

29. Lahne J, Trubek AB, Pelchat ML. Consumer sensory perception of cheese depends on context: a study using comment analysis and linear mixed models. Food Qual Pref 2014;32:184-197.

30. Mascarello G, Pinto A, Parise N, Crovato S, Ravarotto L. The perception of food quality. Profiling Italian consumers. Appetite 2015;89:175-182.

31. Arcia P, Curutchet A, Costell E, Tárrega A. Sensory properties and acceptance of Uruguayan low-fat cheese “queso magro.” Dairy Sci Technol 2013;93:151-62. 